\title{
A Tunable Immitance Simulator with a Voltage Differential Current Conveyor
}

\author{
METIN, B.; ATASOYU, M.; ARSLAN, E.; HERENCSÁR, N.; CICEKOGLU, O.
}

Circuits and Systems (MWSCAS), 2017 IEEE 60th International Midwest Symposium on

pp. 739-742

Electronic ISBN: 978-1-5090-6389-5

Electronic ISSN: 1558-3899

DOI: http://dx.doi.org/10.1109/MWSCAS.2017.8053029

Accepted manuscript

(C2017 IEEE. Personal use of this material is permitted. Permission from IEEE must be obtained for all other uses, in any current or future media, including reprinting/republishing this material for advertising or promotional purposes, creating new collective works, for resale or redistribution to servers or lists, or reuse of any copyrighted component of this work in other works. METIN, B.; ATASOYU, M.; ARSLAN, E.; HERENCSÁR, N.; CICEKOGLU, O., „A Tunable Immitance Simulator with a Voltage Differential Current Conveyor. ", Proceedings of the 2017 IEEE 60th International Midwest Symposium on Circuits and Systems (MWSCAS), pp. 739-742, 2017. DOl: 10.1109/MWSCAS.2017.8053029.

Final version is available at $\mathrm{http}: / /$ ieeexplore.ieee.org/document/8053029 


\title{
A Tunable Immitance Simulator with a Voltage Differential Current Conveyor
}

\author{
Bilgin Metin ${ }^{1 *}$, Mesut Atasoyu ${ }^{2}$, Emre Arslan ${ }^{3}$, Norbert Herencsar $^{4}$, and Oguzhan Cicekoglu ${ }^{5}$ \\ ${ }^{1}$ Dept. of Management Information Systems, Bogazici University, Istanbul, Turkey \\ ${ }^{2}$ Dept. of Electronics Engineering, Istanbul Technical University, Istanbul, Turkey \\ ${ }^{3}$ Dept. of Electronics Engineering, Marmara University, Istanbul, Turkey \\ ${ }^{4}$ Dept. of Telecommunications, Brno University of Technology, Technicka 3082/12, 61600, Brno, Czech Republic \\ ${ }^{5}$ Dept. of Electrical and Electronic Engineering, Bogazici University, Istanbul, Turkey \\ Email: "bilgin.metin@boun.edu.tr
}

\begin{abstract}
In this paper, an electronically tunable immitance circuit is proposed. The presented circuit can be configured as a tunable grounded inductor or capacitor multiplier. The proposed circuit employs a single active element called Voltage Differential Current Conveyor, a single resistor and a single capacitor. The presented circuit does not require element matching constraints. It is linearly tunable over four decades of frequency using bias current control. Simulation results are included to verify theory.
\end{abstract}

Keywords-Current conveyor, inductance simulator, immitance, active filter.

\section{INTRODUCTION}

Immitance simulators have wide application area in analog electronics. They can be used as capacitance multiplier or inductance simulator with suitable choice of passive elements. Immitance simulators may help reducing chip area for example to replace on chip inductors for certain applications. Especially the design of very large capacitors or inductors is impractical in integrated circuit (IC) technology. Some immitance simulator circuits in the literature provide easy electronic tunability if they include current controlled active elements, and this is also exploited in this study.

In the literature many capacitance multiplier circuits have been proposed. Ferri and Pennisi presented a capacitance multiplier circuit by employing a current conveyor, a current operational amplifier, two resistors and a capacitor [1]. Some electronically tunable capacitor multiplier circuits have also been presented in the literature. The circuit in ref. [2] uses two operational transconductance amplifiers (OTA), one operational amplifier, and one capacitor. Abuelma'atti and Tasadduq proposed a circuit using three current conveyors, one resistor and one capacitor [3]. Di Cataldo et. al. presented a circuit using two current conveyors two resistors and one capacitor [4].

Most of the floating or grounded synthetic inductor topologies (Pal [5]-[6], Singh [7], Senani [8], Higashimura and Fukui [9], Paul and Patranabis [10]), suffer from excessive number of active or passive components. The circuits presented in ref. [5-8] use four current conveyors. Another publication presents a circuit suitable for inductor simulation

This study is supported by Bogazici University Research Fund-BAP 10740. Research described in this paper was also financed by the National Sustainability Program under grant LO1401 and by the Czech Science Foundation under grant no. 16-11460Y. For the research, infrastructure of the SIX Center was used. that employs two third generation current conveyors CCIIIs proposed by Liu and Yang [11]. The grounded inductor simulating topology in reference [10] employs only a single current conveyor, four resistors and one capacitor. For each type of inductor at least one condition and/or cancellation constraint is to be satisfied in order to realize the inductor. Moreover for each type of inductor all four passive elements are used. The cancellation constraints require passive component matching which is hardly realizable in practice. Also, Abuelmaatti, Khan, and Al-Zaher [12] proposed floating inductance with only active components.

In this study we proposed an immitance circuit topology providing electronically tunable capacitor multiplier and grounded inductor using a single active element, one resistor and one capacitor. We used a voltage differential current conveyor (VDCC) that is a recently proposed and popular active element [13-18]. The presented topology does not have element matching restriction and employs minimum number of active and passive components. The impedance functions for the nonideal cases are also given. All passive sensitivities are found to be no more than unity. Equations corresponding to these nonidealities are included. Finally a ladder LC highpass filter application example is included.

\section{The VDCC AND PRESENTED CIRCUITS}

The VDCC shown in Fig. 1 is described by the following terminal equations:

$$
V_{n}=V_{p}, \quad I_{z}=I_{n}, \quad I_{x}=g_{m}\left(V_{v}-V_{z}\right), \quad I_{v}=0, I_{p}=0 .
$$

The proposed immitance simulation topology is shown in Fig. 2. The input impedance of the circuit is given in the following equation:

$$
Z_{i}=\frac{2 Y_{1}}{g_{m} Y_{2}}
$$

Asuming $Y_{1}$ and $Y_{2}$ selected as either a capacitor or a resistor the passive sensitivities are no more than unity given as $\left|S_{C}^{Z}\right|=1,\left|S_{G}^{Z}\right|=1$. 


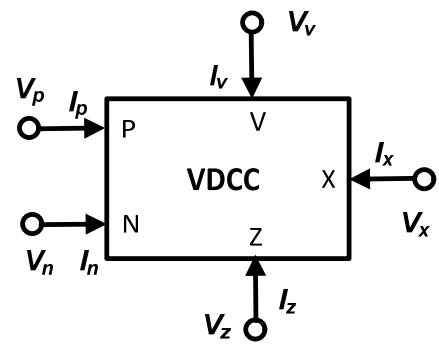

Fig. 1 The VDCC block diagram

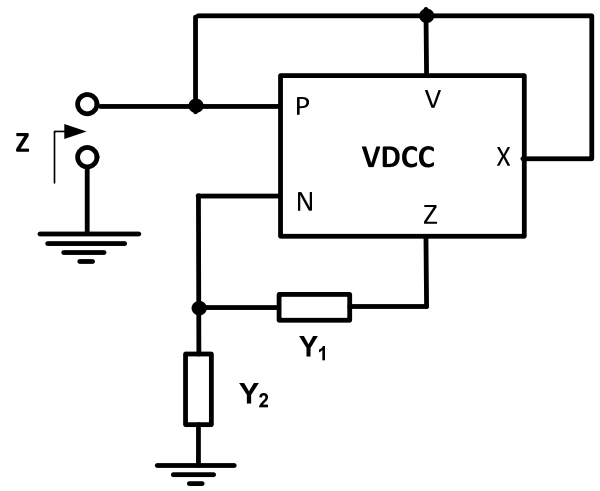

Fig. 2 The presented immitance simulator topology with VDCC

The presented topology in Fig 2 provides a grounded lossless inductor for $Y_{1}=s C$ and $Y_{2}=1 / R$ as shown in Fig. 3a without matching condition and with minimum number of passive components:

$$
L_{e q}=\frac{2 C R}{g_{m}} .
$$

Also a capacitor multiplier can be obtained for $Y_{2}=s C$ and $Y_{1}=1 / R$ as shown in Fig. $3 \mathrm{~b}$ similarly without matching condition and with minimum number of components and a grounded capacitor.

$$
\begin{gathered}
Z_{i}=\frac{1}{s C_{e q}} . \\
C_{e q}=\frac{C R g_{m}}{2} .
\end{gathered}
$$

In the modern MOS IC technology, it is easy to obtain high-resistance values without requiring large chip area using pinched n-well and p-well layer [19]. Although accuracy of this type of resistors is around $25-40 \%$, it is not a disadvantage for tunable circuits since deviation from the center frequency can easily be tuned with an external current.

Inductors are difficult to integrate. Therefore inductance simulators are often used instead. Nonideality analysis is necessary to evaluate the behavior. Taking nonidealities as $V_{n}=\beta V_{p}, I_{\mathrm{z}}=\alpha I_{\mathrm{n}}, I_{\mathrm{x}}=\gamma g_{m}\left(\mathrm{~V}_{\mathrm{v}}-\mathrm{V}_{\mathrm{z}}\right)$, the equivalent nonideal inductance becomes:

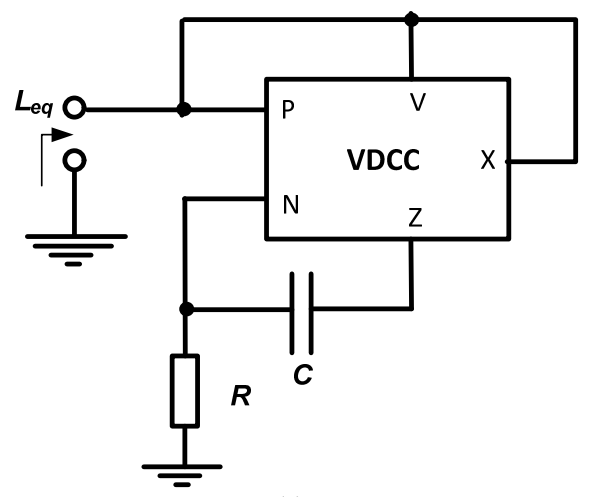

(a)

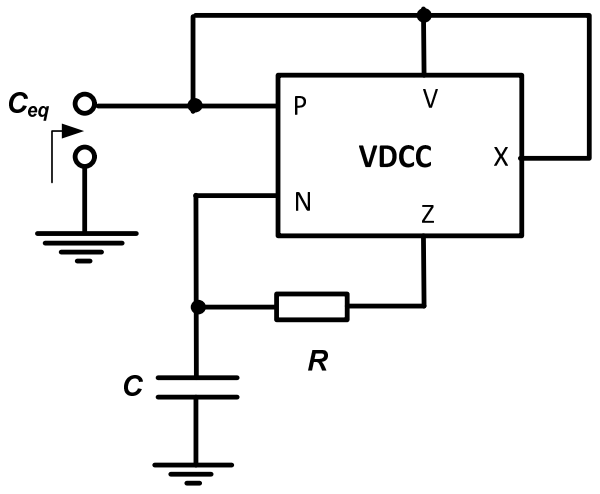

(b)

Fig. 3 The presented circuits: (a) inductance simulator configuration, (b) capacitor multiplier configuration

$$
L_{e q}=\frac{C R(1+\alpha)}{C R g_{m}(1+\alpha)(-1+\beta) \gamma+g_{m} \alpha \beta \gamma} \text {. }
$$

Comparing Eq. 5 with Eq. 3 one can determine how to minimize nonideal effects.

\section{Simulation RESUltS}

The functionality is tested on a third-order Butterworth high-pass prototype LC ladder. In our LTSPICE simulations, a CMOS implementation of VDCC in Fig. 4 is used. The transistor parameters used for the simulations are taken from TSMC $0.35 \mu \mathrm{m}$ technology. The aspect ratios of NMOS and PMOS transistors from the internal CMOS implementation of the VDCC are given in TABLE I. $\mathrm{I}_{0}$ biasing current is $100 \mu \mathrm{A}$. The proposed circuit in Fig. 3a is simulated with the following passive element values: $R=100 \Omega, C=50 \mathrm{pF}$. AC response of the input impedance function of the proposed circuit is given in Fig. 5 for $I_{1}=10 \mu \mathrm{A}$ and $I_{1}=300 \mu \mathrm{A}$. The non-idealities of the VDCC such as current and voltage gain errors, non-ideal output and input impedances of VDCC cause the deviations from theoretical values especially at the low frequencies.

As an application example, a third-order Butterworth highpass ladder filter is chosen as shown in Fig. 6. The component values are: $R_{\mathrm{S}}=R_{\mathrm{L}}=300 \Omega, C_{1}=C_{2}=1 \mathrm{nF}, R=100 \Omega$, $C=50 \mathrm{pF}, \mathrm{I}_{1}=300 \mu \mathrm{A}$ in Fig. 3(a) for $L_{1}=45.5 \mu \mathrm{H}$. The simulation result for the $\mathrm{AC}$ response is shown in Fig. 7. Also 


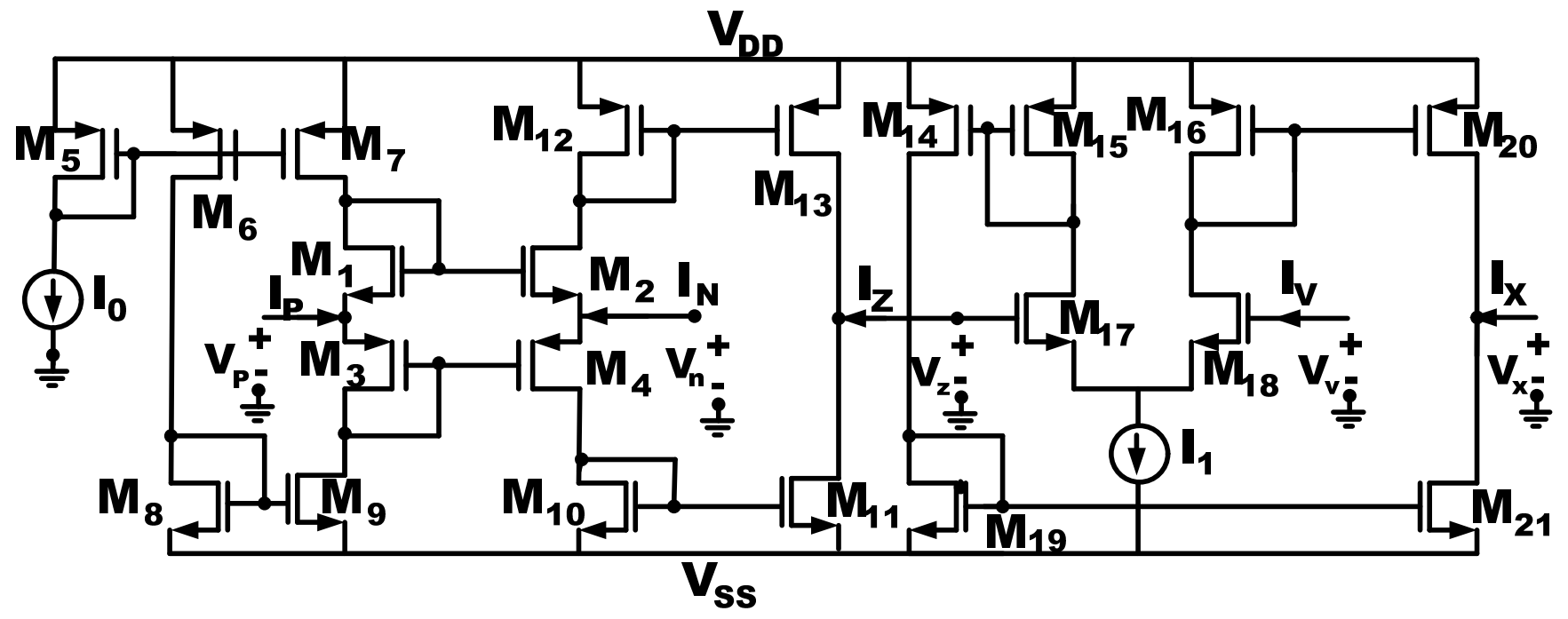

Fig. 4 CMOS implementation of VDCC

TABLE I. TRANSISTOR DIMENSIONS

\begin{tabular}{|c|c|c|c|}
\hline MOSFET & $\mathbf{W}(\boldsymbol{\mu m}) / \mathbf{L}(\boldsymbol{\mu m})$ & MOSFET & $\mathbf{W}(\boldsymbol{\mu m}) / \mathbf{L}(\boldsymbol{\mu m})$ \\
\hline M1 & $20 / 0.35$ & M12 & $30 / 1$ \\
\hline M2 & $20 / 0.35$ & M13 & $30 / 1$ \\
\hline M3 & $60 / 0.35$ & M14 & $8.4 / 1.05$ \\
\hline M4 & $60 / 0.35$ & M15 & $10.5 / 1.05$ \\
\hline M5 & $30 / 2$ & M16 & $10.5 / 1.05$ \\
\hline M6 & $30 / 2$ & M17 & $4.2 / 1.05$ \\
\hline M7 & $30 / 2$ & M18 & $4.2 / 1.05$ \\
\hline M8 & $10 / 2$ & M19 & $4.2 / 1.05$ \\
\hline M9 & $10 / 2$ & M20 & $8.4 / 1.05$ \\
\hline M10 & $10 / 1$ & M21 & $4.2 / 1.05$ \\
\hline M11 & $10 / 1$ & & \\
\hline
\end{tabular}

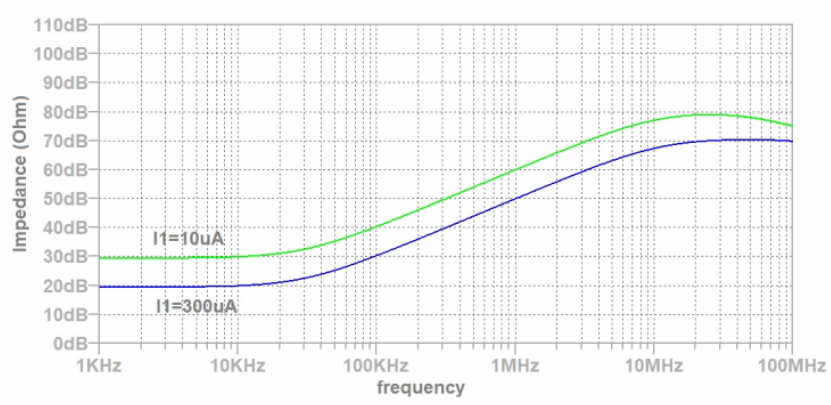

Fig. $5 \mathrm{AC}$ analysis of the inductor simulator configuration (vertical axis represents magnitude of impedance $Z$ with units $\Omega ; 40 \mathrm{~dB}->100 \Omega$ )

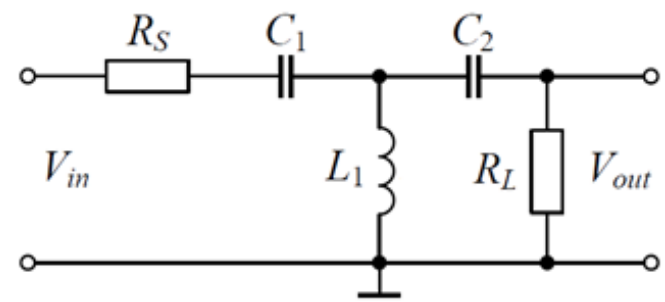

Fig.6 Application example: third-order Butterworth high-pass ladder filter

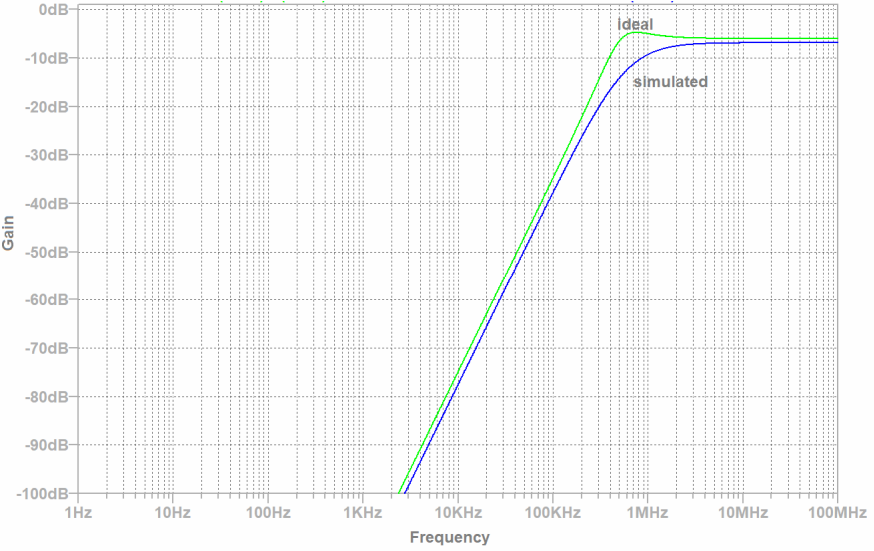

Fig. 7 AC Simulation result of the example filter circuit

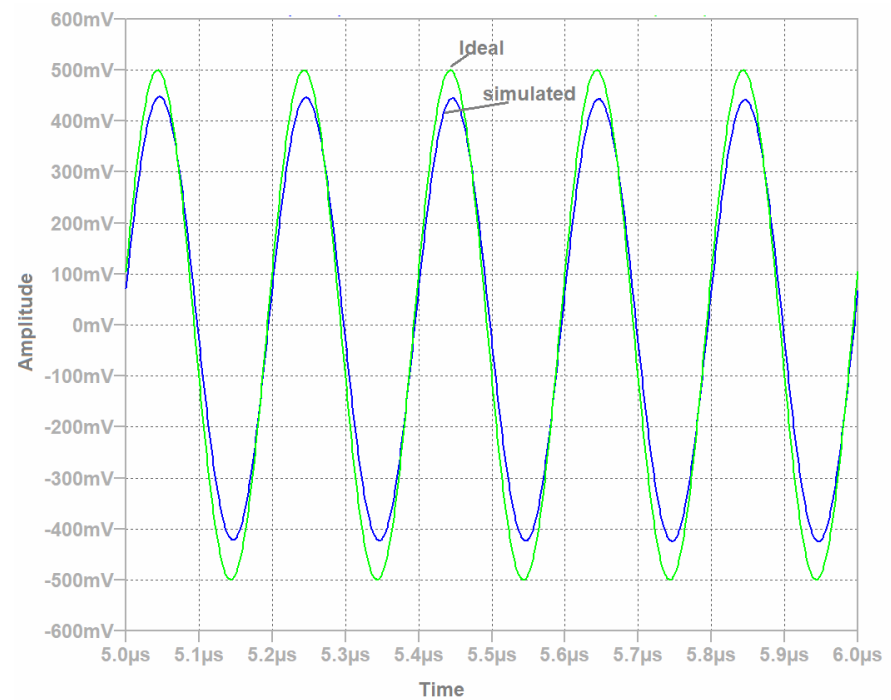

Fig. 8 Transient analysis result of the example filter circuit for $5 \mathrm{MHz}$ output signal (vertical axis represents the output voltage) 
$5 \mathrm{MHz}$ sinusoidal signal with $1 \mathrm{~V}$ peak to peak amplitude is applied to the input of the filter and time domain simulation result is given in Fig. 8. Although some deviations due to the non-ideality of the VDCC, the simulation results confirm well with theory.

\section{CONCLUSION}

In this paper, a grounded immitance simulator topology not requiring passive component matching is proposed. The circuit uses a VDCC, a single capacitor and a single resistor. In the inductance simulator configuration, a high-pass LC ladder filter application example is given to illustrate the practical use of the topology. Simulation results are included to verify theory.

\section{REFERENCES}

[1] G. Ferri and S. Pennisi, "A 1.5V Current-Mode Capacitance Multiplier", Proceedings of the Tenth International Conference on Microelectronics (ICM'98), pp. 9-12, December 14-16, 1998.

[2] M.T. Ahmed, I.A. Khan and N. Minhaj, "Novel electronically tunable Cmultipliers", Electronics Letters, vol. 31 no. 1, 1995.

[3] M.T. Abuelma'atti, N.A. Tasadduq, "Electronically tunable capacitance multiplier and frequency-dependent negative-resistance simulator using the current-controlled current conveyor", Microelectronics Journal, no. 30, pp. 869-873, 1999.

[4] G. Di Cataldo, G. Ferri and S. Pennisi," Active capacitance multipliers using current conveyors", Proceedings of the IEEE International Symposium on Circuits and Systems (ISCAS '98),vol. 2, pp. 343-346, 31 May-3 Jun 1998.

[5] K. Pal, "Novel flotation inductance using current conveyors", Electronics Letters, 1981, 17, no. 18, pp 638.

[6] K. Pal, "New inductance and capacitor floating schemes using current conveyors", Electronics Letters, 1981, 17, no. 21, pp. 807-808.

[7] V. Singh, "Active RC single-resistance-controlled lossless floating inductance simulation using single grounded capacitor", Electronics Letters, 1981,17 , no. 24, pp. 920-921.
[8] R. Senani, "Novel lossless synthetic floating inductor employing a grounded capacitor", Electronics Letters, 1982, 18, no. 10, pp. 413-414.

[9] M. Higashimura, Y. Fukui, "Higher-order immittance function synthesis using current conveyors", Electronics Letters, 1987, 23, no. 10, pp. 498499.

[10] A. N. Paul, D. Patranabis, "Active simulation of grounded inductors using a single current conveyor", IEEE Transactions on Circuits and Systems, 1981, 28, no. 2, pp. 164-165.

[11] S.I. Liu, Y.Y. Yang, "Higher-order immittance function synthesis using CCIIIs", Electronics Letters, 1996, 32, no. 25, pp. 2295-2296.

[12] M.T. Abuelmaatti, M.H. Khan, H. Al-Zaher, "A. Simulation of Activeonly Floting Inductance”, Frequenz, ,1998, vol.52, pp. 161-164.

[13] R. Sotner, J. Jerabek, J. Petrzela, N. Herencsar, R. Prokop, K.Vrba, "Second-order Simple Multiphase Oscillator Using Z-Copy ControlledGain Voltage Differencing Current Conveyor," Elektronika Ir Elektrotechnika, vol. 20, no. 9, pp. 13-18, 2014.

[14] D. Prasad, D.R. Bhaskar, M. Srivastava, "New Single VDCC-based Explicit Current-Mode SRCO Employing All Grounded Passive Components," Electronics, vol. 18, no. 2, pp. 81-88, 2014.

[15] M. Rawat, M. Bansal, "Voltage Mode Two-Phase and Four- Phase Sinusoidal Oscillator Using VDCC," International Journal of Advanced Research in Electronics and Communication Engineering, vol. 4, no. 6, pp. 1687-1691, 2015.

[16] R. Sotner, N. Herencsar, J. Jerabek, R. Prokop, A. Kartci, T. Dostal, K. Vrba, "Z-Copy Controlled-Gain Voltage Differencing Current Conveyor: Advanced Possibilities in Direct Electronic Control of FirstOrder Filter," Elektronika Ir Elektrotechnika, vol. 20, no. 6, pp. 77-83, 2014.

[17] R. Sotner, J. Jerabek, N. Herencsar, T. Dostal, K. Vrba, "Design of Zcopy controlled- gain voltage differencing current conveyor based adjustable functional generator" Microelectronics Journal, vol. 46, no. 2, pp. 143-152, 2015.

[18] R. Sotner, N. Herencsar, J. Jerabek, K. Vrba, T. Dostal, W. Jaikla, B. Metin, "Novel first-order all-pass filter applications of $z$ - copy voltage differencing current conveyor," Indian Journal of Pure and Applied Physics, vol. 53, no. 8, pp. 537- 545, 2015

[19] F. Maloberti, "Analog design for CMOS VLSI Sytems", Kluwer Academic publisher, 2001, pp. 68. 